\title{
Revisiting the multifractality in stock returns and its modeling implications
}

\author{
Shanshan He, Yudong Wang* \\ School of Economics and Management, Nanjing University of Science and \\ Technology, China
}

Xiaolinwei Street 200, Xuanwu District, Nanjing 210094, China

Tel: 8613681663442

* Corresponding author

\section{Acknowledgements}

This work is supported by the Fundamental Research Funds for the Central Universities (No. 30916013106). 


\begin{abstract}
In this paper, we investigate the multifractality of Chinese and the U.S. stock markets using a multifractal detrending moving average algorithm. The results show that stock returns in both markets are multifractal at a similar extent. We detect the source of multifractality and find that long-range correlations are one of the major sources of multifractality in the US market but not in the Chinese market. Fat-tailed distribution plays a crucial role in multifractality of both markets. As an innovation, we quantify the effect of extreme events on multifractality and find the strong evidence of their contribution to multifractality. Furthermore, we investigate the usefulness of popular ARFIMA-GARCH models with skew-t distribution in capturing multifractality. Our results indicate that these models can capture only a fraction of multifractality. More complex models do not necessarily perform better than simple GARCH models in describing multifractality in stock returns.
\end{abstract}

Keywords: MF-DMA; Multifractality; Stock return; Fat-tailed distribution; ARFIMA-GARCH 


\section{Introduction}

The classical Efficient Market Hypothesis (EMH) introduced by Fama [1] is the core of modern finance. However, it is found that many abnormal phenomena cannot be explained by EMH. The fractal market hypothesis (FMH) of Peters [2] is considered as a large challenge to EMH. Peters pointed out that the the fractional Brownian motion were more accurate in depicting the fluctuations of financial asset prices. Fractal time series display many nonlinear characteristics that integer dimension time series do not possess, such as long memory, self-similarity and so on.

Since 1990s, many statistical methods have been developed for fractal analysis. The Rescaled Range Analysis $(\mathrm{R} / \mathrm{S})$, which was proposed by the British water conservancy scientist Hurst [3] in 1951, is one of the most popular methods detecting the long-range correlation of time series. However, R/S produces relatively large estimation error when the series contains short-term memory or the series is not stationary, which often results in the inaccurate estimation of Hurst exponent. In order to overcome the disadvantages of R/S method, Lo [4] proposed the modified R/S method (V/S) to eliminate interference from short-term memory. Nevertheless, R/S and $\mathrm{V} / \mathrm{S}$ methods are only suitable for analyzing the long-range correlation of stationary time series. To overcome the shortcoming of R/S and V/S methods, Peng et al. [5] proposed the detrended fluctuation analysis (DFA) method when studying the self-correlation of DNA sequence. This method is an effective method to quantitatively analyze the long-range correlation of nonstationary time series. Another commonly used method is detrending moving average algorithm (DMA) proposed by 
Alessio et al. [6]. This method replaces the polynomial fitting of DFA method with the moving average and it does not require any assumptions in the estimation of the correlation of the non-stationary time series. The simulating results in Ref. [7-10] show that DMA is better than DFA in all kinds of cases, so it is widely used in the fractal analysis of various time series.

The common drawback of DFA and DMA is that they cannot be used to detect the seminal "stylized fact", multifractality, in financial returns. The multifractal extension of DFA (MF-DFA) of Kantelhardt et al. [11] is a powerful tool for multifractal analysis. Gu and Zhou [12] recently also propose a class of multifractal DMA (MF-DMA) as important extensions of DMA. In this paper, we will use MF-DMA to investigate multifractality in Chinese and the US stock markets.

There are a considerable number of studies focusing on multifractality in financial markets. Notably, Mantegna and Stanley [13] used S\&P 500 indices to re-examine Mandelbrot's finding and found that stock returns actually follow the truncated Levy distribution. The "fat tail" of return distribution shows that the possibility of a huge volatility in financial market is far beyond the normal distribution assumptions. Podobnik [14] used DFA method and found that the stock market of European transition economies displayed the following three characteristics: (1) returns were long-range correlated; (2) volatility had long-range correlation; (3) the return distribution was asymmetric. Qiu [15] made researches on the volatility and trading volume of 18 stocks in Chinese stock market by DFA method, the empirical results of which showed that the volatility and trading volume both had strong long-range 
correlations. Yuan et al. [16] found that the Shanghai Composite index had obvious multifractal characteristics via MF-DFA method and the main cause of multifractality is the long-range correlation. Wang [17-18] analyzed the efficiency of Shanghai stock market using MF-DFA method and found the reform of price limit can reduce the degree of multifractality, therefore improving the efficiency in long term by using a sliding window technique. Zunino [19] found that the Latin American stock market had a high degree of multifractality than the other emerging stock markets. Lu [20] studied the 10-minute trading data of Chinese stock prices, which is characterized by long-term memory and multifractality. Alvarez-Ramirez [21] found that the US stock market became more and more efficient after the collapse of the Breton Woods system by using the time-varying Hurst exponent. Zhuang and Wei [22] found that all the sectoral indices of Chinese stock market exist different degrees of multifractality using the methods of MF-DFA and generalized Hurst exponents. Naser [23] found that DMA approach outperformed any other alternative model used in the forecasting exercise and it provided a better proxy of expected spot prices than future prices. Wang [24] investigated the long-range auto-correlations of crack spreads using MF-DMA method and found that the auto-correlations displayed multiscaling behaviors. There are also many researches on the cause of the multifractality. For example, Norouzzadeh [25], Lee [26] and Kumar [27] analyzed Iranian rial/dollar exchange rate, South Korean stock index and trading volume, and India's financial index, respectively. They found that long-term memory and fat-tailed distribution caused the multifractality. Chen [28] developed the MF-DMA counting technique to 
quantify the components of multifractal spectrum to identify the possible source(s) with the finite-size effect taken into consideration.

In this paper, we focus on the multifractality in Chinese and the US stock markets. In view of the existing literature, the contribution of this paper lies in the following aspects:

First, we study the multifractal behavior of Chinese and the US stock market based on MF-DMA. As previously mentioned, DMA method performs better than DFA in many cases. As a generalization of the DMA, MF-DMA inherits the advantages of DMA and it is more robust and easier to implement with respect to MF-DFA. MF-DMA does not require any assumptions in the estimation of the correlation of nonstationary time series, and it can remove the local trends by subtracting the local means. We found that Chinese and the US stock markets both have apparent multifractal characteristics and the degrees of multifractality are close. The uncertainty of U. S. stock market mainly comes from cumulative risk of small and large fluctuations while the uncertainty of Chinese market is from the influences of extreme events.

Second, most of the existing studies analyze the causes of multifractality only from the dimensions of long memory and fat-tailed distribution. We made further research by investigating the impact of extreme events. We find that long-term memory, fat-tailed distribution and extreme events together lead to the multifractality of the US stock market. Only fat-tailed distribution and extreme events contribute to the multifractality of Chinese stock market. Since the market is immature, the 
contribution of extreme values to multifractality of Chinese stock market is the biggest, while US's lack of controlling of the continuing rising or falling in stock market is responsible for multifractality.

Third, we also quantitatively analyzed the performance of ARFIMA-GARCH model in describing multifractality. Our results indicate that skew-t distribution cannot reflect the real distribution of Shanghai Composite Indice and the Standard \& Poor 500 indice returns. ARFIMA-GARCH models can better describe the multifractal characteristics of the Standard \& Poor 500 indice returns than those of Shanghai Composite Indice returns. More advanced ARFIMA-GARCH models do not necessarily perform better than simple models in capturing multifractality.

The remainder of this paper is organized as follows: The next section provides the methodology. We show data and some descriptive statistics in Section 3. Section 4 shows the empirical results. The modeling implications of multifractality are discussed in Section 5. The last section concludes the paper.

\section{Methodology}

In this paper, we use the recently proposed multifractal detrending moving average (MF-DMA) algorithm to investigate multifractality in stock returns. MF-DMA has been found to outperform traditional MF-DFA and other methods in revealing multifractal properties. For the time series $\left\{x_{t}\right\}, t=1,2, \ldots, N$, where $N$ is the length of the time series, the MF-DMA can be described as follows:

Step 1. Calculate the cumulative values to form a new series,

$$
y(t)=\sum_{k=1}^{t} x_{k}
$$


Step 2. Use the moving window technology to calculate the local means in each moving window,

$$
\widetilde{y}(t)=\frac{1}{n} \sum_{[(n-1) \theta]}^{[(n-1)(1-\theta)]} y(t-k)
$$

where, $n$ is the window size, $\theta$ is the position parameter which takes three values of $0,0.5$ and 1 . Therefore, the independent variables of the moving average function contain $[(n-1)(1-\theta)]$ previous data points and $[(n-1) \theta]$ future data points. In Gu and Zhou [12], they consider three special cases. The first case $\theta=0$ refers to the backward moving average, in which the moving average functions are calculated from the past $n-1$ data points. The second case $\theta=0.5$ refers to the centered moving average, in which the moving average functions are calculated from half past data points and half future data points. The third case $\theta=1$ corresponds to the forward moving average, where functions are calculated from future $n-1$ data points. In this paper, we use $\theta=0$, that is, the backward moving average.

Step 3. Detrend the series by subtracting the moving average functions and get the residual sequence,

$$
\varepsilon(i)=[y(i)-\overline{y(i)}]
$$

where $n-[(n-1) \theta] \leq i \leq N-[(n-1) \theta]$.

Step 4. Divide the residual sequence into $N_{n}$ non-overlapped segments with the length of $n$, where $N_{n}=[N / n-1]$. Each residual series can be written as $\varepsilon_{v}(i)=\varepsilon(l+i), 1 \leq i \leq n$, where $l=(v-1) n$. The fluctuation variance of each sequence can be written as: 


$$
F_{v}^{2}(n)=\frac{1}{n} \sum_{i=1}^{n} \varepsilon_{v}(i)^{2}
$$

Step 5. Calculate the $q$-th order fluctuation function of the sequence. When $q \neq 0$, as the fluctuation function is defined as:

$$
F_{q}(n)=\left\{\frac{1}{n} \sum_{v=1}^{N}\left[F_{v}^{q}(n)\right]\right\}^{1 / q}
$$

When $q=0$, according to L'Hospital's rule, we can get the equation as:

$$
\ln \left[F_{0}(n)\right]=\frac{1}{N_{n}} \sum_{v=1}^{N_{n}} \ln \left[F_{v(n)}\right]
$$

Step 6. Calculate the generalized Hurst exponent $h(q)$ of the fluctuation function according to following power law relationship between $F_{q}(n)$ and $n$ :

$$
F_{q}(n) \propto n^{h(q)}
$$

Step 7. The relationship between $h(q)$ and the quality index $\tau(q)$ can be written as:

$$
\tau(q)=q h(q)-1
$$

Step 8. Calculate the singularity spectrum $f(\alpha)$ according to the quality index $\tau(q)$ :

$$
\alpha=d \tau(q) / d q, f(\alpha)=q \alpha-\tau(q),
$$

where $\alpha$ is the Holder exponent. The singularity spectrum $f(\alpha)$ describes the singularity content of the time series.

\section{Data and descriptive statistics}

We focus on the multifractality property of logarithmic returns. Let $P_{t}$ denote daily closing index at day $t$, then the return $r_{t}$ is obtained by calculating its first 
order logarithmic difference:

$$
r_{t}=\left[\ln \left(P_{t}\right)-\ln \left(P_{t-1}\right)\right]
$$

To construct the returns, we collect the daily closing prices of Shanghai Composite Index (SHCI) and the Standard \& Poor 500 index (S\&P) because Chinese and the US stock markets are important parts of global finance system. SHCI and S\&P 500 are the representatives of indices in emerging and developed markets, respectively. Since Chinese stock market officially starts from December 1990, our sample data cover the period over December 20, 1990 through January 7, 2016, resulting in more than 6000 observations. We obtain the data from Yahoo finance website $^{1}$. Fig. 1 shows the graphical illustrations of stock returns. The volatility of S\&P return series is much lower than that of SHCI during our sample period.

\section{Insert Figure 1 here}

Table 1 displays the descriptive statistics. The average returns of SHCI and S\&P indices are both close to 0 while their sample standard deviations are relatively large. The skewness of the SHCI return is 1.792 , which means that SHCI return series is right-skewed and investors are more likely to gain positive return. The return of S\&P 500 is left-skewed with a skewness of -0.243 , reflecting the larger possibility of investors gaining negative return. We can get that Chinese stock market is relatively more attractive to risk preference investors. To avoid the occurrence of spurious regression, we perform the unit root test on the return series. ADF statistics show the significant rejections of unit root null at $1 \%$ level, indicating the stationarity of returns

\footnotetext{
${ }^{1} \mathrm{http}: / /$ finance.yahoo.com/.
} 
of SHCI and S\&P indices.

\section{Insert Table 1 here}

Fig. 2 shows the probability density functions of stock indices returns. The results in Table 1 and Fig. 2 indicate that both of these series are not subject to normal distribution and they have characteristics of leptokurtosis, especially for SHCI returns. To further investigate the return distribution, we show the quantile-quantile scatterplots (QQ scatterplots) of the stock returns in Fig. 3, using the normal distribution as reference criterion. The returns of SHCI and S\&P indices respectively follow the normal distribution during the intervals $(-0.1,0.1)$ and $(-0.05,0.05)$ while they appear different fat-tail characteristics out of these intervals.

\section{Insert Figure 2 and Figure 3 here}

\section{Empirical results}

4.1 Multifractal analysis of stock returns based on MF-DMA

We set the range of time scale $n$ to be $10<n<N / 10$, where $N$ is the length of each time series. Fig. 4 shows the $\log -\log$ plots of fluctuation functions $F_{q}(n)$ versus time scale $n$ when $q=-10,-2,2$ and 10 for the both return series. We can observe apparent power-law relationships between $F_{q}(n)$ and $n$ for each value of $q$.

\section{Insert Figure 4 here}

We use the generalized Hurst exponents $h(q)$ and local Holder index $\alpha$ to describe the multifractal characteristics of stock indices returns. Fig. 5 shows the values of the generalized Hurst exponents $h(q)$ (denoted by the legend "original series") when $q$ varies from $[-10,10]$. Overall, the values of $h(q)$ are negatively 
related to $q$, indicating that SHCI and S\&P indices return series are strongly multifractal.

\section{Insert Figure 5 here}

Fig. 6 displays the multifractal spectra of $\alpha \sim f(\alpha)$ via the Legendre transform (denoted as the legend "original series"). The inverse parabolic shapes of spectra confirms the fact of multifractality in Chinese and the US stock market. Interestingly, we find the negative fractal dimension $f(\alpha)$ for S\&P returns. Mandelbrot [29] considers that the positive dimensions $f(\alpha)$ are shown to define a "typical" distribution of the measure, while a negative $f(\alpha)$ determines the sampling variability. In our case, the negative dimension can be observed in the left parts of the spectra implying that more noise in S\&P returns may be caused by large fluctuations.

\section{Insert Figure 6 here}

\subsection{Origins of multifractality}

It has been well documented in the literature that there are two major origins of multifractality, long-range correlations of small and large fluctuations and fat-tailed distribution [25-27]. In this section, we will innovatively consider the effect of a third factor, extreme events, on the multifractal property. We investigate the contribution of long-range correlations by comparing the multifractality of the original series and randomly shuffled series. The procedure of obtaining the shuffled series can be described as follows:

(1) Generate a random pair of natural numbers $(p, q)$ with $p, q$ not bigger than the length of time series, $N$. 
(2) Exchange the $p$-th and $m$-th data in the original sequence.

(3) Repeat the steps above $20 \mathrm{~N}$ times to ensure the original series be fully shuffled.

We can compare the multifractality between the original series and surrogated series to investigate the contribution of fat-tailed distribution. We follow Zhou [30] in using the following method to generate surrogated series with normal distribution:

(1) For a given series, we can generate a series of random numbers with Gaussian distributions $\left\{r_{t}^{\prime}, t=1, \ldots \ldots, N\right\}$ after discrete Fourier transform.

(2) Rearrange the series generated in (1) to get the rearranged series $\left\{r_{t}, t=1, \ldots \ldots, N\right\}$ which has the same rank ordering as the original series.

To quantify the effects of extreme values (EV) on the multifractality of SHCI and S\&P indices returns, we get the EV removed series using the method as follows.

(1) Get a new series $\left\{y_{t}, t=1, \ldots \ldots, N\right\}$ by sorting the original return series $\left\{x_{t}, t=1, \ldots \ldots, N\right\}$. Remove the first $T / 2$ and the last $T / 2$ data points from $\left\{y_{t}, t=1, \ldots \ldots, N\right\}$ and get a new series $\left\{z_{t}, t=1, \ldots \ldots, N-T\right\}$ without EV points where $T$ is the number of the data points in the tail of the series. Here, we define $T=5 \% * N$.

(2) Replace the first $T / 2$ and the last $T / 2$ data points in $\left\{y_{t}, t=1, \ldots \ldots, N\right\}$ with data points randomly chosen from $\left\{z_{t}, t=1, \ldots \ldots, N-T\right\}$. Then we obtain a series $\left\{y_{t}^{\prime}, t=1, \ldots \ldots, N\right\}$ without extreme values, the length of which is $N$.

(3) Generate the EV removed series $\left\{x_{t}^{\prime}, t=1, \ldots \ldots, N\right\}$ which has the same rank order with original series $\left\{x_{t}, t=1, \ldots \ldots, N\right\}$ by rearranging series $\left\{y_{t}^{\prime}, t=1, \ldots \ldots, N\right\}$. Fig. 5 also displays how the generalized Hurst exponents $h(q)$ vary with $q$ for 
the shuffled, surrogated and EV removed series. The generalized Hurst exponents $h(q)$ display negative relationships with $q$ while the EV removed series of SHCI returns is an exception since its generalized Hurst exponents $h(q)$ for positive values of $q$ is larger than those for negative values of $q$. This abnormal phenomenon indicates that extreme events account for the increasing volatility of Chinese stock market. The corresponding multifractal spectra are also given in Figure 6.

The above analysis is only a roughly qualitative determination of multifractal. For more detailed information, we use $\Delta \alpha$ and $\Delta h$ to quantify the degree of multifractality.

$$
\begin{aligned}
& \Delta \alpha=\alpha_{\max }-\alpha_{\min } \\
& \Delta h=h(q)_{\max }-h(q)_{\min }
\end{aligned}
$$

Table 2 shows the multifractality degrees of the original, shuffled, surrogated and EV removed series of SHCI and S\&P indices returns. Comparing multifractality degrees between SHCI and S\&P returns, we can find that the multifractality degrees of the two original return series are rather close. S\&P return series has larger $\Delta \alpha$, displaying higher degree of multifractality.

For S\&P indices return series, the multifractality degrees of shuffled series, surrogated series and EV removed series are smaller than those of original series indicating that long-range correlations, fat-tailed distribution and extreme events are all contribute to the multifractality. Fat-tailed distribution has the biggest contribution to multifractality, followed by extreme events. The multifractality degree is still relatively higher than zero after removing extreme values, indicating that the risk 
cumulated by persistent small increases and decreases account for the uncertainty of the US stock market.

For SHCI return series, similarly, fat-tailed distribution and extreme events lead to the multifractality. The impact of extreme events is larger than that of fat-tailed distribution since its $\Delta h$ is much lower. Chinese stock market appears a high level of immaturity caused by the implementation of unreasonable policies, frequent regulation and irrational investor behavior. All these factors contribute to the high uncertainty of stock market. The $\Delta h$ and $\Delta \alpha$ of the shuffled series are much close to those of original series, indicating that long-range correlations have little effect on multifractality. Thus, the uncertainty of Chinese stock market mainly comes from extreme events.

\section{Insert Table 2 here}

\section{Modeling implications}

In this section, we discuss the ability of popular econometric models in capturing multifractality in stock returns. We consider the models accommodating three important sources of multifractality, long-range correlations, fat-tailed distribution and extreme volatility. The autoregressive fractional integrated moving average (ARFIMA) model has been widely employed to account for long-range correlations. The empirical distribution of financial asset returns is considered to be close to the skewed student $\mathrm{t}$ distribution. GARCH-class models are the powerful tool for modeling and forecasting volatility dynamics after the seminal work of Engle [31]. Based on these 
motivations, we use the ARFIMA-GARCH-Skew-t models. Our purpose is to verify whether it can effectively describe the multifractal characteristics or reflect the real distribution of return after accounting for three contributions of mutifractality in the econometric models. We compare the multifractality degrees between original and ARFIMA-GARCH-implied standardized residuals. The lower multifractality degree in the residuals implies that the related model can better capture multifractal behavior in stock returns. The specifications of the models under consideration are given as follows.

\subsection{Autoregressive Fractionally Integrated Moving Average (ARFIMA) model}

The ARFIMA model approved by Granger and Joyeux [32] and Hosking [33] is definitely powerful tool that can portray long memory. For a time series $\left\{x_{t}\right\}$, $t=1,2,3, \ldots \ldots, T, \operatorname{ARFIMA}(r, d, s)$ model can be expressed as:

$$
\begin{gathered}
\Phi(L)(1-L)^{d}\left(x_{t}-\mu\right)=\Theta(L) \varepsilon_{t} \\
\varepsilon_{t}=z_{t} \sigma_{t}
\end{gathered}
$$

where $\mu$ is the conditional mean, $\Phi(L)$ and $\Theta(L)$ are, respectively, $r$ and $s$ order polynomial, which take the forms as $\Phi(L)=\phi_{1} L+\phi_{2} L^{2}+\ldots+\phi_{r} L^{r}$ and $\Theta(L)=\theta_{1} L+\theta_{2} L^{2}+\ldots+\theta_{s} L^{s}$. We compare two distributions of $z_{t}$, normal distribution and Skew-t distribution. The density function of skew-t distribution proposed by Hansen [34] is given by: 


$$
g(z \mid \eta, \lambda)=\left\{\begin{array}{l}
b c\left(1+\frac{1}{\eta-2}\left(\frac{b z+a}{1-\lambda}\right)^{2}\right)^{-(\eta+1) / 2}, z<-a / b, \\
b c\left(1+\frac{1}{\eta-2}\left(\frac{b z+a}{1+\lambda}\right)^{2}\right)^{-(\eta+1) / 2}, z \geq-a / b
\end{array}\right.
$$

where $2<\eta<\infty$ and $-1<\lambda<1$. The parameters $a, b$ and $c$ are given by:

$$
a=4 \lambda c\left(\frac{\eta-2}{\eta-1}\right), b^{2}=1+3 \lambda^{2}-a^{2} \text { and } c=\frac{\Gamma\left(\frac{\eta+1}{2}\right)}{\sqrt{\pi(\eta-2)} \Gamma\left(\frac{\eta}{2}\right)}
$$

When $\lambda=0$, skew-t distribution was the same with traditional student $t$ distribution. Apparently, ARFIMA reduces to ARMA $(r, s)$ model when $d=0$, which represents the short memory process. It is called non-stationary ARIMA $(r, s)$ model while $d=1$. When $d$ varies from 0 to 1 , equation (13) denotes a long memory process. Especially, it can be divided into long memory stationary process or long memory non-stationary process according to whether the parameter $d$ is greater or less than 0.5 .

\subsection{ARCH-class models}

ARCH-class models are widely used to describe and forecast time-varying volatility of financial assets in the area of financial econometrics. We use following GARCH-class models:

The first volatility model is the simple $\operatorname{GARCH}(1,1)$ developed by Bollerslev [35], which is an extension of Engle [31]'s ARCH model. The specification of GARCH $(1,1)$ can be expressed as: 


$$
\sigma_{t}^{2}=\omega+\alpha \varepsilon_{t-1}^{2}+\beta \sigma_{t-1}^{2},
$$

where $\sigma_{t}^{2}$ is conditional variance. GARCH model needs to impose non-negative constraints on parameters, such as $\omega>0, \alpha \geq 0, \beta \geq 0$, to guarantee $\sigma_{t}^{2}>0$. The stationarity condition of volatility is $\alpha+\beta<1$.

Nevertheless, financial assets display asymmetric volatility, which reflects different impacts caused by good news and bad news. Notably, Tenenbaum et al. [36] analyze eleven European transition economies and find that like developed economies, almost all transition economy indices exhibit a significant volatility asymmetry. Furthermore, they find that developed and transition economies experience different association between volatility and volatility asymmetry. ARCH and GARCH models themselves cannot portray the dynamics of volatility resulting from the up and down of returns. For this consideration, we use the GJR model [37]. The specification of GJR model can be expressed as:

$$
\sigma_{t}^{2}=\omega+\left[\alpha+\gamma I\left(\varepsilon_{t-1}<0\right)\right] \varepsilon_{t-1}^{2}+\beta \sigma_{t-1}^{2},
$$

where $I($.$) is the indicator function. That is to say, we assign its value of 1$ when it meets the condition in the parenthesis and zero otherwise. Likewise, GJR model needs to add non-negative constraint on parameters such as $\omega>0, \alpha \geq 0, \beta \geq 0$, to guarantee the non-negative value of $\sigma_{t}^{2}$. The parameter $\gamma$ is used to describe the asymmetry of fluctuation and the stationary condition is $\alpha+\beta+\gamma / 2>0$.

The aforementioned two GARCH models assume that the autocorrelation of volatility decays at exponential rates, i.e., short memory. Since the long memory in volatility has been considered a seminal "stylized fact", we use the fractionally 
integrated GARCH (FIGARCH) [38]. The FIGARCH $(1, d, 1)$ can be described as:

$$
\sigma_{t}^{2}=\omega+\beta \sigma_{t-1}^{2}+\left[1-(1-\beta L)^{-1}(1-\varphi L)(1-L)^{d}\right] \varepsilon_{t}^{2}
$$

The non-negative conditions of FIGARCH is $0 \leq d \leq 1, \omega>0, \varphi, \beta<1$. The fractional integration parameter $d$ describes the property of long memory in volatilities and $L$ is the lag operator. The conditional volatility follows the long memory process when $0<d<1$.

Since the FIGARCH does not accommodate asymmetry, we use the fractionally integrated FIEGARCH [39] allowing for both asymmetric effect and long memory in volatilities. The specification of $\operatorname{FIEGARCH}(1, d, 1)$ is given by:

$$
\begin{aligned}
& h_{t}=\left(1-L^{d}\right)\left(\ln \sigma_{t}^{2}-\omega\right)=\beta h_{t-1}+g\left(z_{t-1}\right)+\varphi g\left(z_{t-2}\right), \\
& g\left(z_{t}\right)=\theta z_{t}+\gamma\left(\left|z_{t}\right|-E\left|z_{t}\right|\right) .
\end{aligned}
$$

\subsection{On the usefulness of models}

We generate standardized residuals from ARFIMA-GARCH, ARFIMA-GJR, ARFIMA-FIGARCH and ARFIMA-FIEGARCH models. Each model is based on Gaussian and skew-t distributions. Therefore, we can obtain 8 residual series for SHCI or S\&P returns.

Table 3 and Table 4 show the multifractality degrees of model filtered standardized residuals for Shanghai and US stock markets, respectively. For S\&P returns, the multifractality degrees of residuals are approximately equal to 0.1 and much lower than those of original series. However, for SHCI returns, the multifractality degrees of residuals are closer to those of original series. This evidence 
suggests that ARFIMA-GARCH model can well capture multifractality in the US stock markets but its performance in capturing multifractality in Chinese markets is relatively poor.

Insert Table 3 and Table 4 here.

For SHCI or S\&P return series, the differences of multifractality degrees between model filtered standardized residuals based on skew-t distribution and those based on Gaussian distribution is very minor. This result indicates that skew-t distribution cannot reflect the real distributions although it theoretically takes fat-tail characteristics into account.

The multifractality degrees of residuals from GJR-GARCH model and FIGARCH model are close to GARCH model. That is, asymmetry and long memory in volatilities are not the main cause for the multifractality. Thus, more complex volatility models cannot better capture multifractal characteristics.

\section{Conclusions}

In this paper, we investigate the multifractality of returns of Shanghai Composite indices and the Standard \& Poor 500 indices using the multifractal detrending moving average algorithm. We find that SHCI and S\&P indices return series are multifractal at a similar extent evidenced by the changes of the generalized Hurst exponents $h(q)$ over the fluctuation orders $q$ and the inverse parabolic shapes of multifractal spectra. On the origins of multifractality, the main sources of multifractality in the US stock market are long-range correlations, fat-tailed distribution and extreme events. The contribution of fat-tailed distribution is the greatest, followed by the extreme events. 
The risk cumulated by sustained increases or decreases in stock prices may account for the uncertainty of the US stock market. As to SHCI return series, fat-tailed distribution and extreme events, especially the latter one, lead to the multifractality but the effect of long-range correlations is very minor. Thus, the uncertainty of Chinese stock market mainly comes from extreme changes in stock prices due to some events such as the implementation of government policy and irrational investor behaviors.

As an innovation, we investigate the ability of advanced econometric models in capturing multifractality stock returns. We use the ARFIMA-GARCH models accommodating three "stylized facts" of stock returns, long memory, volatility dynamics and fat-tailed distributions. We find that these models can well describe multifractality of S\&P returns but its performance in depicting multifractality of SHCI returns is very poor. According to the multifractal analysis of these series based on the MF-DMA method, our results show that Skew-t distribution cannot reflect the real distribution of SHCI or S\&P indices daily return. More sophisticated ARFIMA-GARCH models do not necessarily perform better than simple ones in characterizing multifractality behavior.

\section{References}

[1] E. Fama, Efficient capital markets: A review of theory and empirical work, Journal of Finance 25 (1970) 383-417.

[2] E. Peter, Chaos and order in the capital market, John Wiley \& Sons, New York, 
1991.

[3] H.E. Hurst, Long-term storage capacity of rserviors, Transactions of the American Society of Civil Engineers 116 (1951) 770-808.

[4] A.W. Lo, Long-term memory in stock market prices, Econometrica 59 (1991) 1279-1313.

[5] C.K. Peng, S.V. Buldyrev, S. Havlin, M. Simon, H.E. Stanley, A.L. Coldberger, Mosaic organization of DNA nucleotides, Physical Review E 49 (1994) 1685-1689.

[6] E. Alessio, A. Carbone, G. Castelli, V. Frappietro, Second-order moving average and scaling of stochastic time series, Eur. PHYS. J.B 27 (2002) 197-200.

[7] L.M. Xu, P.C. Ivanov, K. Hu, Z. Chen, A. Carbone, H.E. Stanley, Phys.Rev.E $71(2005) 051101$.

[8] A. Caebone, G. Castelli, H.E. Stanley, Time-dependent Hurst exponent in financial time series, Physica A 344 (2004) 267-271.

[9] R. Matsushita, I. Gleria, A. Figueiredo, S.D. Silva, Are pound and euro the same currency? Physics Letters A 368 (2007) 173-180.

[10] A. Serletis, A.A. Rosenberg, Mean reversion in the US stock market,Chaos, Solitons \& Fractals 40 (2009) 2007-2015.

[11] J.W. Kantelhardt, S.A. Zschiegner, E. Koscienlny-Bunde, S. Havlin, A. Bunde, H.E. Stanley, Multifractal detrended fluctuation analysis of nonstationary time series, Physica A 316 (2002) 87-114.

[12] G.-F. Gu, W.-X. Zhou, Detrending moving average algorithm for multifractals, Physical Review E 82 (2010) 011136. 
[13] R.N. Mantegna, H.E. Stanley, Scaling behavior in the dynamics of an economic index, Nature 376 (1995) 46-49.

[14] B. Podobnik, D. Fu, T. Jagric, I. Grosse, H.E. Stanley, Fractionally integrated process for transition economics, Physica A 362 (2006) 465-470.

[15] T. Qiu, L.X. Zhong, X.R. Wu, Statistical properties of trading volume of Chinese Stocks, Physica A 388 (2009) 2427-2434.

[16] Y. Yuan, X.T. Zhuang, X. Jin, Measuring multifractality of stock market price fluctuation using multifractal detrended fluctuation analysis, Physica A 388 (2009) 2189-2197.

[17] Y. Wang, Y. Wei, C. Wu, Detrended fluctuation analysis on spot and futures markets of West Texas Intermediate crude oil, Physica A 390 (2011) 864-875.

[18] Y. Wang, C. Wu, Z. Pan, Multifractal detrending moving average analysis on the US Dollar exchange rates, Physica A 390 (2011) 3512-3523.

[19] L. Zunino, B.M. Tabak, A. Figliola, P.G. Perez, M. Garavaglia, O.A. Rosso, A multifractal approach for stock market efficiency, Physica A 387 (2008) $6558-6566$.

[20] X. Lu, J. Tian, Y. Zhou, Z. Li, Multifractal detrended fluctuation analysis of the Chinese stock index futures market, Physica A 392 (2013) 1452-1458.

[21] J. Alvarez-Ramirez, J. Alvarez, E. Rodriguez, G. Femandez-Ananya, Timevarying Hurst exponent for US stock markets, Physica A 387 (2008) 6159-6169.

[22] X. Zhuang, Y. Wei, Multifractality, efficiency analysis of Chinese stock market and its cross-correlation with WTI crude oil price, Physica A 430 (2015) 101-113. 
[23] Hanan Naser, Estimating and forecasting the real prices of crude oil: A data rich model using a dynamic model averaging (DMA) approach, Energy Economics 56 (2016) 75-87.

[24] Y. Wang, C. Wu, What can we learn from the history of gasoline crack spreads?: Long memory, structural breaks and modeling implications, Economic Modelling 29 (2012) 349-360.

[25] P. Norouzzadeh, B. Rahmani, A multifractal detrended fluctuation description of Iranian rial-US dollar exchange rate, Physica A 367 (2006) 328-336.

[26] K.E. Lee, J.W. Lee, Probability distribution function and multiscaling properties in the Korean stock market, Physica A 383 (2007) 65-70.

[27] S. Kumar, N. Deo, Multifractal properties of the Indian financial market, Physica A 388 (2009) 1593-1602.

[28] F. Chen, K. Tian, X. Ding, Y. Miao, C. Lu, Finite-size effect and the components of multifractality in transport economics volatility based on multifractal detrending moving average method, Physica A 462 (2016) 1058-1066.

[29] B.B. Mandelbrot, Negative fractal dimensions and multifractals, Physica A 163 (1990) 306-315.

[30] W. Zhou, The components of empirical multifractality in financial returns, Europhysics Letters 88 (2) 28004.

[31] R.F. Engle, Autoregressive conditional heteroskedasticity with estimates of the variance of United Kingdom inflation, Econometrica 50 (1982) 987-1007.

[32] C.W.J. Granger, R. Joyeux, An introduction to long-memory time series models 
and fractional differencing, Journal of Time Series Analysis 1 (1980) 15-39.

[33] J.R.M. Hosking, Fractional differencing, Biometrika 68 (1981) 165-176.

[34] B. E. Hansen, Autoregressive conditional density estimation.,International Economic Review 35 (1994) 705-730.

[35] T. Bollerslev, Generalized autoregressive conditional heteroskedasticity, Journal of Econometrics 31 (1986) 307-327.

[36] J. Tenenbaum, D. Horvatic, S. C. Bajić, H. E. Stanley, Comparison between response dynamics in transition economies and developed economies, Physical Review E 82 (2010) 046104.

[37] L.R. Glosten, R. Jagannathan, D.E. Runkle, On the relation between the expected value and the volatility of the nominal excess return on stocks, Journal of Finance 48 (1993) 1779-1801.

[38] R.T. Baillie, T. Bollerslev, H.O. Mikkelsen, Fractionally integrated generalized autoregressive conditional heteroskedasticity, Journal of econometrics 74 (1996) $3-30$.

[39] T. Bollerslev, H.O. Mikkelsen, Modeling and Pricing Long Memory in Stock Market Volatility, Journal of Econometrics 73 (1996) 151-184. 
Figures

SHCI

S\&P
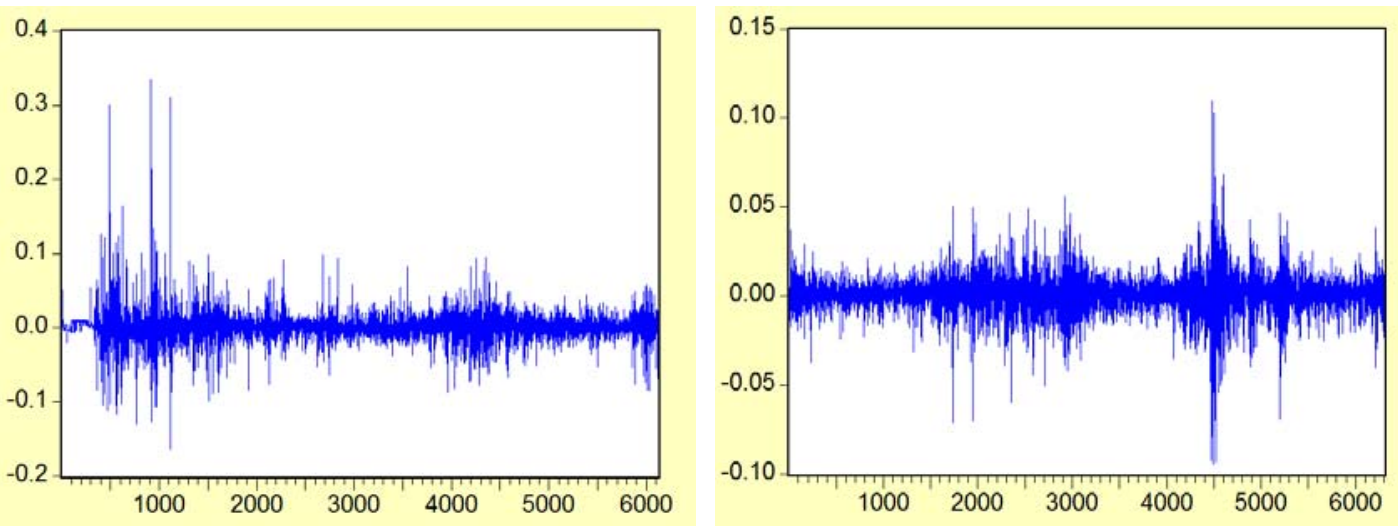

Fig. 1. Stock return series. 
SHCI

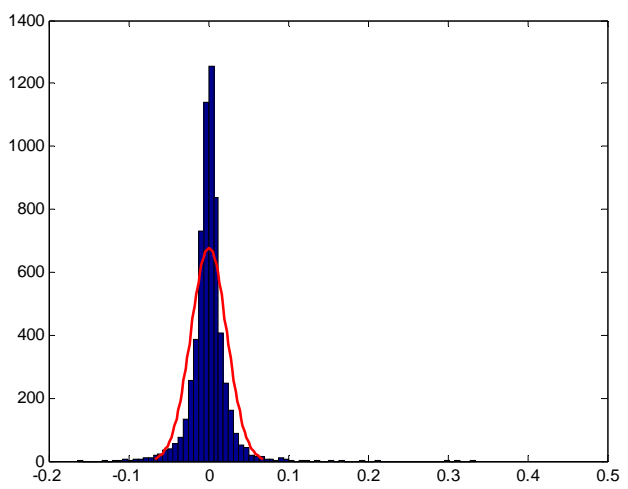

S\&P

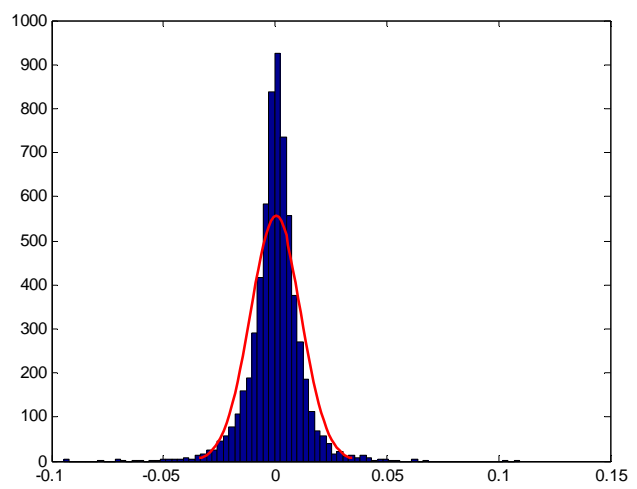

Fig. 2. Probability density functions of stock indices returns. 

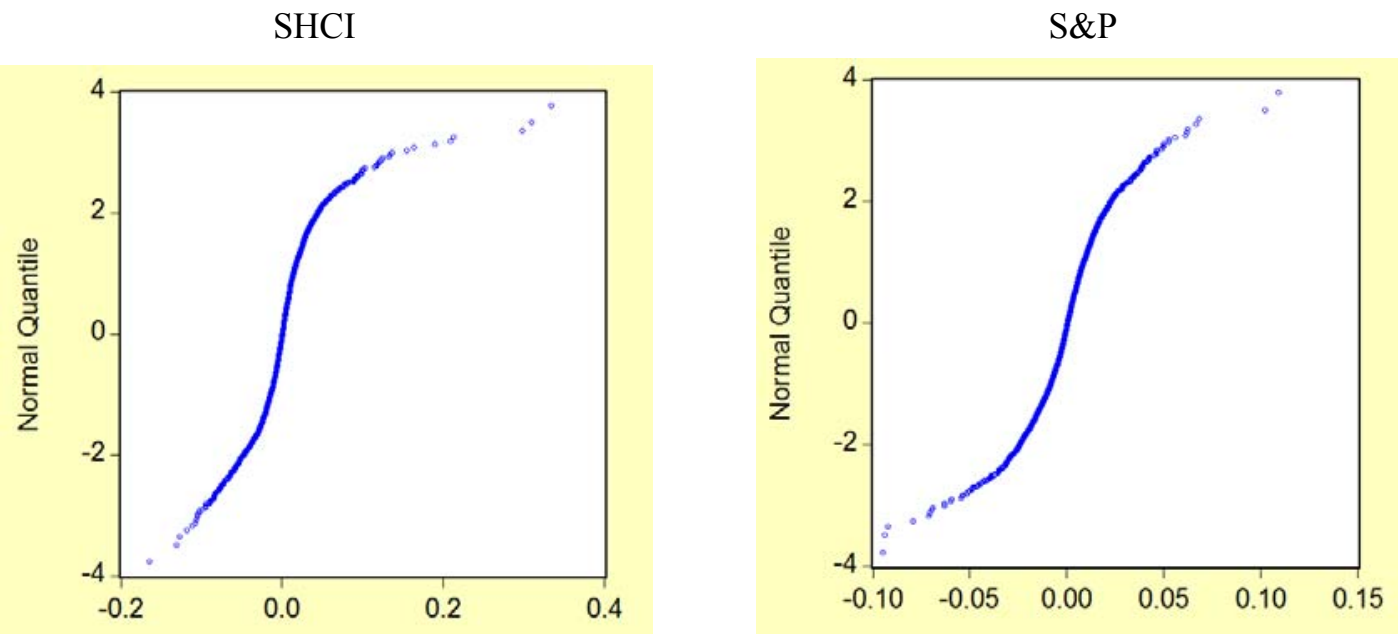

Fig. 3. Quantile-quantile scatter plots of stock returns. 
SHCI

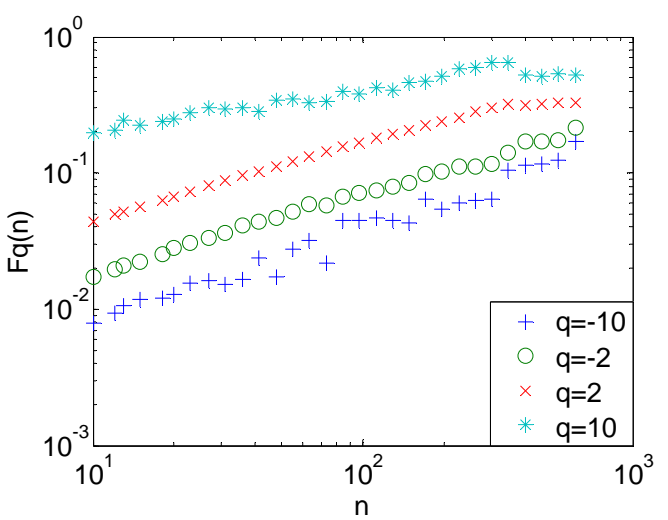

S\&P

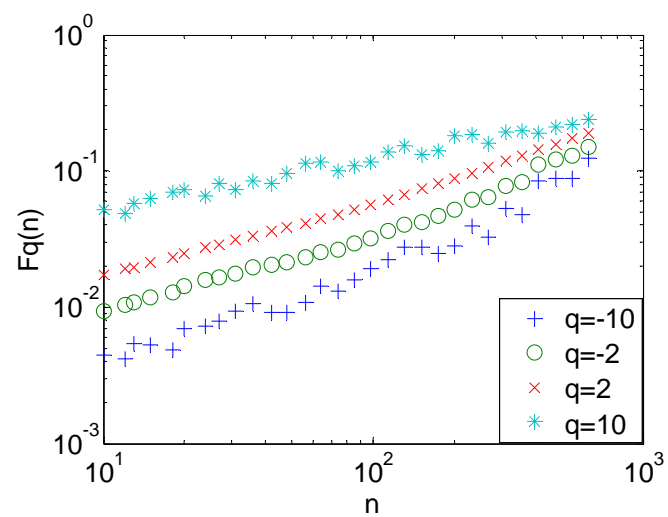

Fig. 4. $\log -\log$ plots of fluctuation functions $F q(n)$ versus time scale $n$. 
SHCI

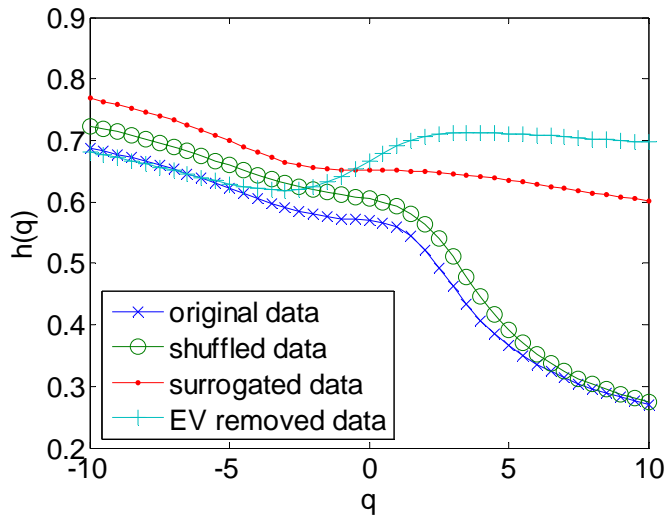

S\&P

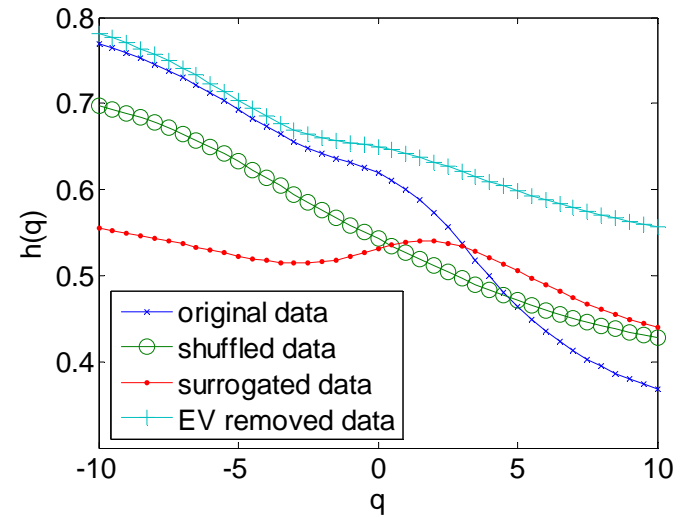

Fig. 5. Generalized Hurst exponents of SHCI and S\&P indices return series based on MF-DMA. 
SHCI

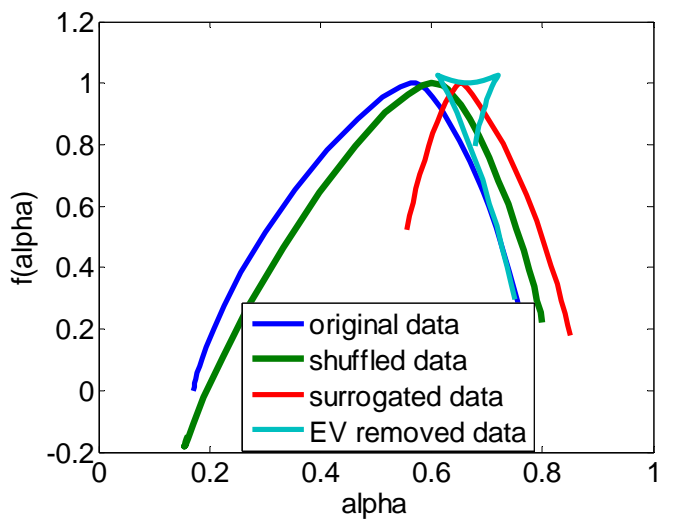

S\&P

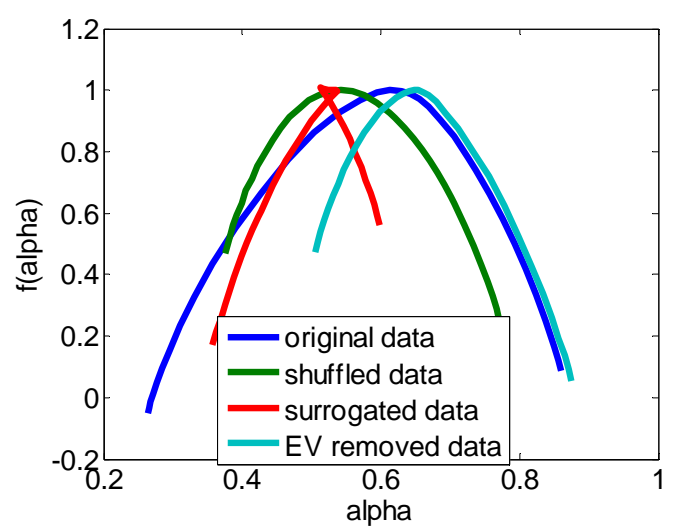

Fig. 6. Multifractal spectra calculated by the Legendre transform. 


\section{Tables}

Table 1

Descriptive statistics.

\begin{tabular}{ccc}
\hline & SHCI & S\&P \\
\hline No. of observations & 6127 & 6311 \\
Mean & $6.92 \mathrm{e}-4$ & $2.81 \mathrm{e}-4$ \\
Median & $7.00 \mathrm{e}-4$ & $5.21 \mathrm{e}-4$ \\
Maximum & 0.335 & 0.110 \\
Minimum & -0.164 & -0.095 \\
Std. dev & 0.022 & 0.011 \\
Skewness & 1.792 & -0.243 \\
Kurtosis & 30.328 & 11.809 \\
JB & $193938.400^{* * *}$ & $20467.410^{* * *}$ \\
ADF & $-30.9680^{* * *}$ & $-38.445^{* * *}$ \\
\hline
\end{tabular}

Note: The Jarque-Bera (JB) statistic tests for the null hypothesis of normality in sample returns distribution. ADF are statistics of the Augmented Dickey-Fuller unit root test in which the optimal lag lengths are determined based on the AIC criterion .

The asterisks $* * *$ and $* * *$ denote rejections of null hypothesis at $10 \%, 5 \%$ and $1 \%$ significance levels, respectively. 


\section{Table 2}

Multifractality degrees of four series.

\begin{tabular}{ccccccccc}
\hline & \multicolumn{4}{c}{$\Delta h$} & \multicolumn{5}{c}{$\Delta \alpha$} \\
\cline { 2 - 9 } & Original & Shuffled & Surrogated & EV removed & Original & Shuffled & Surrogated & EV removed \\
\hline SHCI & 0.416 & 0.448 & 0.166 & 0.094 & 0.593 & 0.645 & 0.294 & 0.138 \\
S\&P & 0.402 & 0.270 & 0.116 & 0.225 & 0.597 & 0.400 & 0.241 & 0.370 \\
\hline
\end{tabular}


Table 3

Multifractality degrees of standardized residuals for SHCI returns.

\begin{tabular}{lllllllll}
\hline & \multicolumn{7}{c}{ Gaussian distribution } & \multicolumn{3}{c}{ Skew-t distribution } \\
\cline { 2 - 9 } & GARCH & GJR & \multicolumn{2}{c}{ FIGARCH } & FIEGARCH GARCH & GJR & FIGARCH FIEGARCH \\
\hline$\Delta h$ & 0.307 & 0.283 & 0.268 & 0.323 & 0.314 & 0.271 & 0.301 & 0.387 \\
$\Delta \alpha$ & 0.477 & 0.451 & 0.428 & 0.484 & 0.501 & 0.448 & 0.472 & 0.577 \\
\hline
\end{tabular}




\section{Table 4}

Multifractality degrees of standardized residuals for S\&P returns.

\begin{tabular}{lllllllll}
\hline & \multicolumn{7}{c}{ Gaussian distribution } & \multicolumn{2}{c}{ Skew-t distribution } \\
\cline { 2 - 9 } & GARCH & GJR & \multicolumn{2}{c}{ FIGARCH } & FIEGARCH GARCH & GJR & \multicolumn{2}{c}{ FIGARCH FIEGARCH } \\
\hline$\Delta h$ & 0.079 & 0.114 & 0.066 & 0.068 & 0.070 & 0.099 & 0.062 & 0.095 \\
$\Delta \alpha$ & 0.097 & 0.136 & 0.095 & 0.147 & 0.098 & 0.124 & 0.114 & 0.173 \\
\hline
\end{tabular}

